\title{
In memoriam Professor Dr. med. Heinrich Jungbluth
}

In memoriam Professor Dr. med. Heinrich Jungbluth

\section{Bibliografie}

DOI http://dx.doi.org/ 10.1055/s-0029-1244157 Pneumologie 2010; 64: 390-391 ๔ Georg Thieme Verlag KG Stuttgart · New York ISSN 0934-8387

Korrespondenzadresse Dr. Robert Kropp

Liegnitzer Straße 5 36100 Petersberg dr.robert.kropp@gmx.de
Am 7. März 2010, in seinem 88. Lebensjahr, starb Herr Professor Dr. med. Heinrich „Henner“ Jungbluth nach langer Krankheit. Er wurde am 16. Januar 1923 in Monzingen/Nahe geboren; formende Jugendjahre erlebte er in Mailand - daher stammen seine Liebe zu Italien und seine exzellenten Kenntnisse der italienischen Sprache und als Soldat in Russland und Italien. Nach dem Weltkriege studierte er Humanmedizin in Pisa und Frankfurt am Main; hier bestand er 1951 sein medizinisches Staatsexamen und wurde im selben Jahr zum Doktor der Medizin promoviert. Anschließend war Heinrich Jungbluth als Volontär, Assistent, schließlich als Oberarzt an der ehemaligen II. Medizinischen Universitäts-Klinik und -Poliklinik in Frankfurt am Main tätig. Dort arbeitete er auf verschiedenen Stationen, so auf einer Abteilung für Tumorkranke und auf der Tuberkuloseabteilung, und erwarb umfangreiches Wissen und einen großen Fundus an Erfahrungen, nicht nur im Bereich der Inneren Medizin. Sein beruflicher Werdegang wurde von Persönlichkeiten wie den Professoren Joachim Frey, Max Gänsslen, Helmut Martin und Karl-Ludwig Radenbach geprägt. Auf der Tuberkulosestation lernte er die ersten wirksamen Medikamente gegen die Tuberkulose kennen; vor allem Isoniazid weckte damals sein Interesse. Heinrich Jungbluth veröffentlichte in den folgenden Jahren zahlreiche wissenschaftliche Arbeiten, vor allem über die Chemotherapie der Tuberkulose. $\mathrm{Zu}$ erwähnen sind auch seine weiteren Publikationen, zum Beispiel über die Diagnostik der Nierentuberkulose, über zytostatische Therapien; das Kapitel über die Therapie der Tuberkulose im Handbuch der Inneren Medizin wurde von ihm mitverfasst. 1970 habilitierte er sich an der Medizinischen Fakultät der JohannWolfgang-Goethe-Universität in Frankfurt am Main mit der Arbeit „Die Elimination des Isonikotinsäure-Hydrazids und seiner Metaboliten aus dem Plasma bei Kranken mit eingeschränkter Nierenfunktion“.

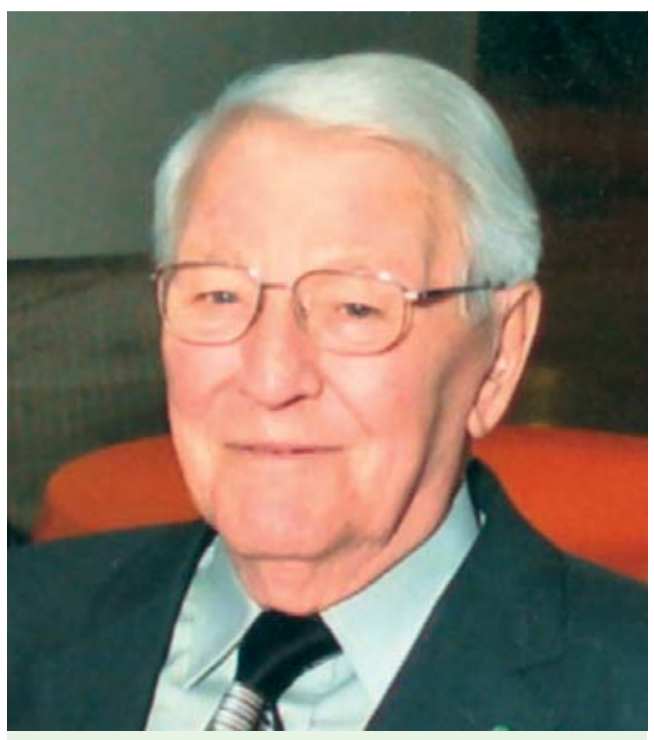

Professor Dr. med. Heinrich Jungbluth.

1970 übernahm Heinrich Jungbluth als ärztlicher Direktor die Leitung der LVA-Klinik Seltersberg in Gießen. Diese erste und lange Zeit einzige Heilstätte für Lupuskranke gestaltete er nach und nach von einer reinen Tuberkuloseheilstätte um in eine moderne Klinik für Lungen- und Bronchialerkrankungen mit Diagnostik und Therapie unspezifischer und spezifischer sowie auch maligner bronchopulmonaler Krankheiten. Im Jahre 1974 wurde er vom Fachbereich Humanmedizin der Justus-Liebig-Universität Gießen zum Honorarprofessor berufen.

Professor Jungbluth war 1967 Mitbegründer der Paul-Ehrlich-Gesellschaft für Chemotherapie. Er war Mitglied und Vorstandsmitglied der wissenschaftlichen Arbeitsgemeinschaft für die Therapie von Lungenkrankheiten (WATL). Während dieser Zeit beteiligte er sich an zahlreichen kooperativen, kontrollierten Studien zur Wirksamkeit kombinierter antituberkulöser Chemotherapie; hieraus entstand schließlich die Kurzzeittherapie 
der Tuberkulose. Er wurde in den Vorstand der Sektion „Pneumologie“ der Akademie für ärztliche Fort- und Weiterbildung der Landesärztekammer Hessen in Bad Nauheim berufen. Lange Jahre war er maßgeblich im Versorgungswerk der Landesärztekammer Hessen tätig.

1990 war er einer der ersten, der nach der deutschen Wiedervereinigung den Kontakt zu den Pneumologen vor allem in Thüringen fand.

Für seine Verdienste erhielt Heinrich Jungbluth die Dr.-RichardHammer-Medaille, 1997 die Ernst-von-Bergmann-Plakette der Bundesärztekammer. 1991 wurde er Ehrenmitglied der Süddeutschen Gesellschaft für Pneumologie, später Ehrenmitglied der Mitteldeutschen Gesellschaft für Pneumologie. Hervorzuheben ist weiterhin, dass er für seine Verdienste als langjähriger Vertrauensarzt des italienischen Konsulats in Frankfurt am Main mit dem Ritterkreuz des Verdienstordens der Italienischen Republik (Cavaliere dell“ Ordine „Al Merito della Repubblica Italiana“) geehrt wurde.

Heinrich Jungbluth war ein gebildeter, kluger und vielseitig interessierter, fröhlicher Mensch. Für viele ältere und jüngere Kollegen war er Vorbild und ein gesuchter Ratgeber. Er liebte die (italienische) Küche, einen guten Wein, die Musik. Wir verlieren mit ihm einen Freund und eine Persönlichkeit, auf die wir uns stets verlassen konnten. Unser Mitgefühl gilt vor allem seiner Ehefrau und seinen Kindern mit Angehörigen. Wir werden ihn im Gedächtnis behalten. 